Marcela Natalia Arango*, Carlos Mario Rodríguez R.**, Andrea Carolina Fonseca ***, Fernanda Lisseth Pachón ${ }^{* * * *}$, Diana Camila Cuervo *****

\title{
Pasos de Libertad: Propuesta transmedia para el fortalecimiento de la apropiación del valor patrimonial ${ }^{1}$
}

Pasos de Libertad: Transmedia proposal to strengthen the appropriation of heritage value

Cómo citar:

Arango, M., Cuervo, D., Fonseca, A., Pachón, F. \& Rodríguez C. M. (2019). Pasos de libertad. Propuesta transmedia para el fortalecimiento de la apropiación del valor patrimonial. Designia, 6(2), 9-23. ${ }^{1}$ Este artículo presenta la propuesta ganadora de la convocatoria
Crea Digital 2018 de los ministerios de Cultura y de Tecnologías de
Información y las Comunicaciones de Colombia. Con los recursos
obtenidos mediante este reconocimiento se financia parcialmente
la ejecución del proyecto, gestado y desarrollado en su fase inicial
desde el grupo de investigación Xisqua del programa de Diseño
Gráfico, en alianza con el semillero de formación en investigación

GAMA de la Universidad de Boyacá.

*Docente investigadora del Grupo Xisqua de la Universidad de Boyacá, Diseñadora Gráfica, Magister en Docencia Universitaria.

E-mail: mnarango@uniboyaca.edu.co ORCID: http://orcid.org/0000-0002-3357-6511

**Docente investigador del Grupo Xisqua de la Universidad de Boyacá, Diseñador Gráfico, Magister en Entornos Virtuales de Aprendizaje.E-mail: carrodriguez@uniboyaca.edu.co ORCID: https://orcid.org/0000-0002-5670-7393

***Docente investigadora del Grupo Xisqua de la Universidad de Boyacá, Comunicadora Social, Magister en Administración de Organizaciones. E-mail:andcarfonseca@uniboyaca.edu.co ORCID: https://orcid.org/0000-0002-0811-1755

**** Estudiante del Semillero Gama de la Universidad de Boyacá. Diseñadora Gráfica. E-mail: flpachon@uniboyaca.edu.co ORCID: https://orcid.org/0000-0002-6918-7753

***** Estudiante del Semillero Gama de la Universidad de Boyacá. Diseñadora Gráfica. E-mail: dccuervo@uniboyaca.edu.co ORCID: https://orcid.org/0000-0003-4823-7114

\section{Palabras clave:}

Patrimonio, ecosistema digital, transmedia.

Key words:

Heritage, digital ecosystem, transmedia.

Recibido: 31/08/2018

Aceptado: 29/01/2019 


\section{Resumen:}

El presente artículo de reflexión da cuenta de una propuesta para la implementación de Contenidos Educativos Digitales a través de narrativas transmediales que acerquen a las nuevas generaciones a temas como la Ruta Libertadora en Boyacá. El problema de investigación se plantea a partir de la reflexión respecto a la manera de fortalecer y divulgar el valor patrimonial y cultural de dicha Ruta en entornos no convencionales que cumplan una función educativa. Se presentan los desarrollos realizados tras el análisis y diagnóstico de la comunidad escolar en el colegio Silvino Rodríguez de la ciudad de Tunja, a quienes se destina inicialmente un prototipo correspondiente a un lugar de relevancia como es el Puente de Boyacá. Este prototipo incluye elaboración de guion, redacción de contenidos, doblaje de voces, grabación de video, animación, creación de personajes, diagramación, producción de una aplicación móvil y un libro electrónico (e-book). De tal modo, se busca reducir la brecha digital y dinamizar este tipo de contenidos, hacerlos más accesibles y responder a las necesidades detectadas en el trabajo realizado con los estudiantes.

\section{Abstract:}

This paper reflects on a proposal aiming to implement digital educational content through transmedial narratives that engage new generations in subjects such as the Ruta Libertadora (Route of Freedom) in Boyacá. The research problem deals with strengthening and disseminating the heritage and cultural value of this Route in unconventional environments for educational purposes. Following the analysis and diagnosis of a student community attending the Silvino Rodríguez School in Tunja, a prototype has been worked out and its development is described here. Focusing on the historical site Puente de Boyacá (Bridge of Boyacá), it is targeted at the students and includes script, content adaptation, voice dubbing, video recording, animation, character creation, a mobile application and an e-book. It is thus intended reducing the digital gap, fostering this type of content, making it more accessible and responding to the needs identified when working with the School's young population. 


\section{INTRODUCCION}

Las tecnologías avanzan constantemente, cambian la cultura y la forma de vida de las personas. Así, a través de la historia se han logrado avances y progresos en las sociedades, se han transformando problemáticas en soluciones creativas, muchas veces con protagonismo de lo tecnológico. En nuestro tiempo, un reto radica en la implementación de las Tecnologías de Información y Comunicación (TIC) en la cotidianidad. Además, si permitimos que estas se vinculen a procesos de enseñanza y aprendizaje, se posibilita un escenario para que las nuevas generaciones las apropien y hagan parte de su diario vivir, con lo cual se reducen las brechas digitales.

En esta propuesta, una acción enmarcada en un proceso educativo es promover y fortalecer, con el apoyo de las TIC, la apropiación del patrimonio tangible de una región que es parte de la cultura histórica del país. De tal modo, nos hacemos responsables como instituciones educativas de la labor de sensibilizar a esas nuevas generaciones frente a elementos patrimoniales y de identidad nacional.

Con el fin de delimitar el grupo objetivo destinatario de la propuesta a desarrollar, la investigación realizada vinculó a la comunidad educativa del Instituto Integrado Nacional Silvino Rodríguez de la ciudad de Tunja. De esta manera, se estableció que se espera llegar a jóvenes entre ocho y doce años, estudiantes de grados cuarto a sexto. Cabe subrayar que el mencionado centro educativo, como muchos otros en Colombia, imparte la cátedra de Historia nacional pero frecuentemente carece de herramientas didácticas facilitadoras de su tarea. En consecuencia, el proyecto aborda dicha problemática y plantea una posible respuesta a la misma.

Como parte del trabajo de sensibilización y acercamiento con la comunidad escolar, se efectuaron talleres, encuestas y entrevistas con estudiantes, docentes y directivos. Esta información permitió definir la pertinencia de una estrategia transmedia basada en Contenidos Educativos Digitales (CED) que incluya un libro electrónico (e-book), un mapa interactivo y material audiovisual con descargas gratuitas, todo vinculado a una aplicación móvil y una página web. De igual forma, se constató que las competencias a fortalecer en los escolares son: lectoescritura, tecnología, apropiación del conocimiento e identidad cultural. 


\section{CONCEPTUALIZACIÓN DE LA PROPUESTA}

La generación de CED para esta propuesta se relaciona con la necesidad de socializar los resultados investigativos obtenidos por el grupo PAME (Patrimonio y Memoria) de la Facultad de Arquitectura, Diseño y Urbanismo de la Universidad de Boyacá en el tema de la Ruta Libertadora. Si bien este ha sido un riguroso trabajo, se ha detectado que carece de difusión y no llega a contextos educativos, por lo tanto, se espera vincularlo a la comunidad, especialmente escolar.

Adicionalmente, en los colegios se ha evidenciado la ausencia de material educativo gratuito y de fácil acceso que favorezca una efectiva apropiación del patrimonio por parte de los jóvenes. En este contexto, el grupo Xisqua identificó una oportunidad para enlazar la investigación de PAME con CED transmedial que pueda proyectarse para responder a necesidades educativas de los colegios en dicha materia.

Desde una fundamentación conceptual, ya que las nuevas tecnologías posibilitan la difusión del conocimiento, cabe ejecutar acciones orientadas a vincular estos recursos con las nuevas generaciones y permitirles servir como herramientas en la preservación del patrimonio, pues este se enfrenta a realidades cambiantes que exigen estrategias pensadas de acuerdo con las demandas de cada momento.

Es importante, entonces, promover acciones que aporten a esas demandas y permitan reflexionar en torno al patrimonio no solo como un concepto sino como aquello que brinda sentido, identidad y pertenencia a una comunidad. Se presenta así una oportunidad para minimizar el distanciamiento hacia bienes con valor cultural e histórico, los cuales ameritan ser conocidos y cuidados.

Para abordar la producción de material digital, es preciso reconocer que millones de personas, particularmente niños y jóvenes, viven inmersas en teléfonos inteligentes, videojuegos, computadoras y redes sociales. Al respecto, Iriarte (2011) afirma:

Estamos frente a otro tipo de niño, producto no de una determinada concepción de infancia que tenía en cierto momento la sociedad como era antes, sino un niño producto de una sociedad tecnológica, que por principio no es neutra. Desde pequeñitos comienzan a interactuar con los medios informáticos (p. 221). 
Estos medios ofrecen múltiples horizontes. En algunos casos pueden poseer poco contenido educativo, razón por la cual se reafirma la pertinencia de generar CED con narrativas transmediales que respalden procesos escolares y de apropiación patrimonial. Como sugieren Cope y Kalantzis (2009), se trata de incluir las nuevas tecnologías en métodos de enseñanza tradicional. Ciertamente, en la actualidad estas herramientas tecnológicas son un medio adecuado para llegar a las nuevas generaciones y acercar distintas temáticas de forma didáctica e innovadora.

\title{
ANTECEDENTES
}

El proyecto se desprende del macroproyecto "Resignificación patrimonial de la Ruta Libertadora en Boyacá" y de su publicación Por los caminos de la Ruta Libertadora (Mesa, Millares, Guerrero, Jiménez, \& Bonilla, 2009), en la cual se busca reconstruir la memoria colectiva de los municipios que hacen parte de dicha Ruta y reivindicar la historia de la campaña emancipadora de 1819 en el marco de la conmemoración de su bicentenario en 2019. Para la propuesta aquí descrita se retomaron dichos contenidos y se buscó presentarlos de forma innovadora y coherente con el grupo objetivo. Además, hacerlo mediante tecnologías y narrativas transmediales pueda considerarse como otra contribución a la conmemoración. Se ha comprendido que

\begin{abstract}
La era digital amplió las posibilidades de los medios tradicionales, que han pasado a coexistir como un complemento. Esta conjunción ha posibilitado actividades interactivas y atractivas, orientadas a estimular el interés de los estudiantes por los contenidos tratados, tanto en el aula como en casa. Los profesores en sus asignaturas suelen ahora usar libros digitales como herramienta alternativa no tradicional. Esto multiplica las posibilidades de los procesos educativos, propicia otras formas de acceder a la información, fortalece el conocimiento y puede mejorar el rendimiento académico. (Martínez, Pinzón \& Arango, 2017, p. 61).
\end{abstract}

Con esta orientación se han realizado varios trabajos, desde productos existentes en el mercado literario infantil hasta experiencias investigativas. Cabe destacar:

LuaBooks, empresa colombiana, que ha innovado con los llamados libros transmedia. Estos se diferencian del multimedia en ser una forma de narración a través de diferentes plataformas que permiten interacción con un libro impreso, una aplicación, un juego, o un cortometraje (Carvajal, 2013). 
El proyecto pedagógico Editep (Aparicio, 2005) de la Universidad de Cuyo, Argentina, brindó educación a agentes de la policía de la provincia de Mendoza en una modalidad semipresencial que implementó las TIC como herramienta principal.

El objetivo de la investigación de Cano (2009) fue favorecer una mayor identificación con elementos patrimoniales por medio de la educación. El trabajo, realizado con gabinetes pedagógicos de Bellas Artes e instituciones dedicadas a la producción de materiales, buscó la difusión entre los ciudadanos del patrimonio. Para Cano, este "es pasado pero también es compromiso con el presente, y las nuevas tecnologías pueden ser un medio eficaz de 'anclaje' con este presente y con la construcción de nuestra identidad, en contacto con otros tiempos y otros lugares" (p. 134).

Rascón y Sánchez (2008) valoran de manera crítica las posibilidades de las tecnologías y cómo estas ayudan a la comprensión de sitios históricos. Su propuesta, una colaboración en que tecnología y didáctica van de la mano, consta de una réplica 3D de una excavación y un modelado de objetos arqueológicos.

La revisión y caracterización de estos trabajos permitió reconocer recursos, tales como aplicaciones, páginas web, multimedios, enciclopedias virtuales, videojuegos, e-books y narraciones transmediales factibles de aplicarse en procesos educativos para facilitar la participación del estudiante usuario. En la investigación para la propuesta transmedial a construir se consideró necesario comparar y delimitar los alcances y las limitaciones de cada herramienta en aras de identificar aquella que cumpliera con los requerimientos del contexto y grupo estudiantil objetivo.

Los contenidos producidos pueden afianzar un aprendizaje heutagógico, es decir, aquel en que el estudiante es responsable de su propio proceso sin depender de las lecciones impartidas en una clase formal. De tal forma, se evita reproducir la concepción predominante en varios casos, consistente en usar las TIC tan solo como medio proveedor de información y no como mediador didáctico orientado a garantizar la autogestión del aprendizaje (Aguiar, Velázquez \& Aguiar, 2019). Esta ha sido una premisa en la conceptualización del proyecto.

Las experiencias previas reseñadas sirvieron para definir un camino hacia la creación de una propuesta que intenta articular contenido educativo, cultural y gráfico al generar una aplicación móvil, un e-book de descarga gratuita, material audiovisual, animaciones y la inclusión complementaria de realidad virtual. 
Al constatar la viabilidad de producir CED para responder a la problemática identificada en el colegio Silvino Rodríguez, se reconoce en los contenidos transmediales una forma didáctica de aprender y acceder al conocimiento. Se tiene claro que estas narrativas poseen la facultad de ser flexibles y constituir, de manera conjunta con otras herramientas, un sistema de aprendizaje más integral.

Es importante resaltar, a su vez, que "las interfaces funcionan en doble vía pues acercan la información a los usuarios a la vez que estos se acercan a la información" (Rodríguez, 2015, p. 13). Por tal razón, el diseño debe estar pensado dentro de un proceso de interacción y realimentación para integrar toda la propuesta a partir de la gráfica. En este orden de ideas y al tener claro el grupo objetivo (jóvenes de instituciones educativas), se consideró procedente articular un equipo interdisciplinario con la vinculación de diseñadores, comunicadores, pedagogos e ingenieros de sistemas, entre otros profesionales, para la producción del prototipo.

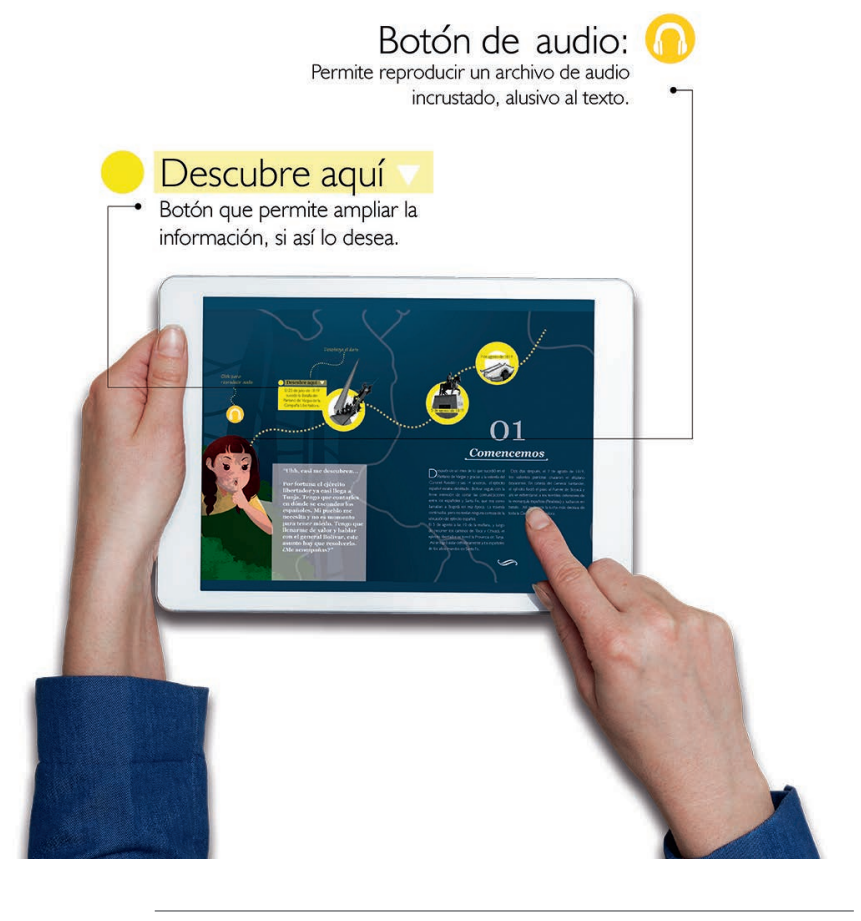

Figura 1. Diseño de una de las páginas del e-book. Fuente: autores (disponible en https://adobe.ly/2J2Jhbb). 
A continuación, se presenta, de manera sucinta, la etapa de planeación y desarrollo inicial del primer prototipo, correspondiente al volumen titulado "Puente Boyacá". El proceso realizado comprende el concepto del proyecto, su funcionalidad y la descripción de su estructura, al igual que la síntesis de la descripción narrativa.

- Planeación. Esta fase abarca: selección de contenidos, redacción y línea editorial del libro (historia de la Ruta Libertadora, personajes y sitios históricos, guía de acceso para docentes y alumnos), guion, arquitectura de información y contenidos.

- Desarrollo y producción. Incluye: propuesta de diseño y de personajes, edición fotográfica, maquetación, diagramación, animación, arreglos y doblaje de voces.

- Propuesta transmedia. Una vez se tiene la línea editorial y gráfica, se desarrollan el e-book, la página web, el demo del aplicativo móvil y la estructura de la aplicación. Todo esto muestra el recorrido planteado y destaca el valor del Puente de Boyacá.

\section{Elementos de diseño integrados}

Marca. "Pasos de Libertad", es la marca a gran escala que reúne los lugares por donde transcurrió la Ruta Libertadora. Su logotipo está acompañado por charreteras, elementos de los uniformes de la guardia presidencial de la Gran Colombia. (Ver figura 2).

Retícula. Para lograr una lectura adecuada, se diseñó una retícula que organiza los distintos elementos (texto, imágenes, botones, entre otros). Se procura así brindar orden y legibilidad al lector mientras avanza por las páginas. (Ver figura 3).

Tipografía. Como fuentes tipográficas se seleccionaron Georgia y Humanist. La primera fue diseñada por Matthew Carter en 1996, especialmente para ser leída en pantallas. La segunda, una versión de la Gill Sans creada por Eric Gill en 1931, facilita la lectura de corrido al tener menos remates, ya que su estilo humanista con proporciones clásicas le confiere una singular apariencia. (Ver figura 4).

Fotografías. Se incorporan con el propósito de acercar al usuario a los escenarios y personajes reales (patrimonio tangible), en particular a los monumentos construidos en honor a los sucesos que llevaron a la victoria de las tropas patriotas. (Ver figura 5).

Tlustraciones. Como fuerza expresiva del texto, acercan a los niños y jóvenes al contenido escrito. Cuentan con un estilo simplificado, funcional e infográfico. (Ver figura 6). 


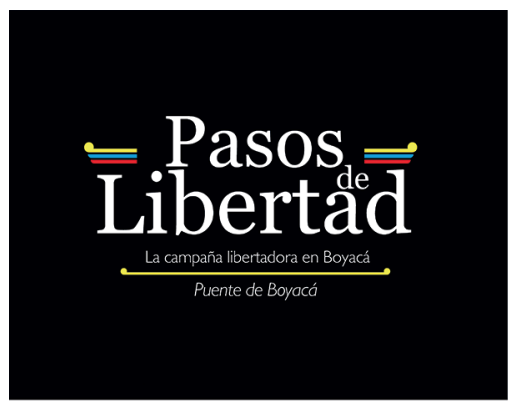

\section{$\overline{\overline{\mathrm{L}}} \operatorname{Pasos}_{\mathrm{d}} \overline{\bar{d}}$ + Libertaded \\ La campaña libertadora en Boyać Puente de Boyacá}
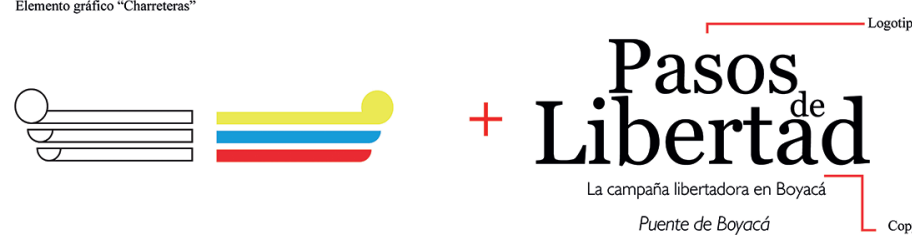

Figura 2. Diseño de marca. Fuente: autores.

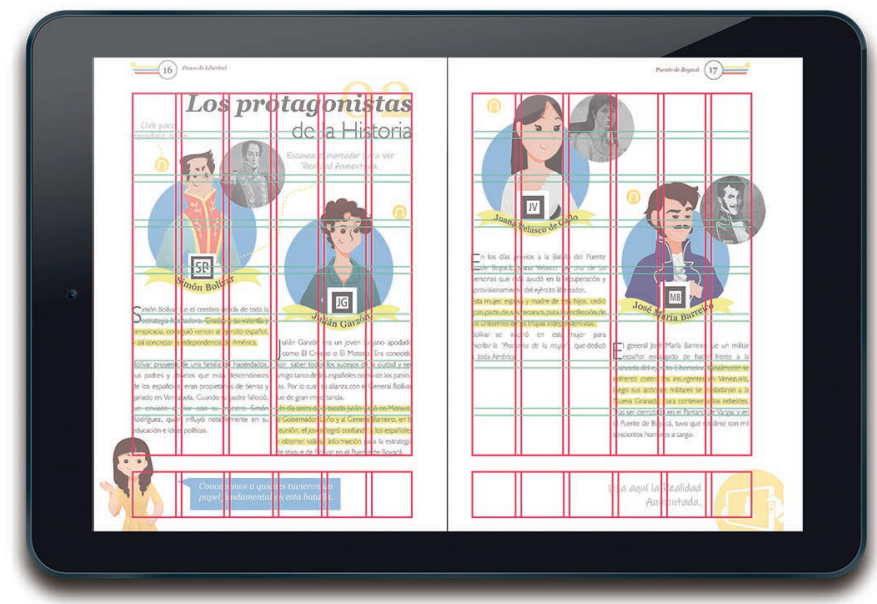




\section{Georgia}

Regular | Italic | Bold | Bold Italic The five boxing wizards jump quickly.

\section{Humanist 52I Regular | Italic | Bold | Bold Italic}

Light | Light Italic

The five boxing wizards jump quickly

Figura 4. Fuentes tipográficas. Fuente: autores.
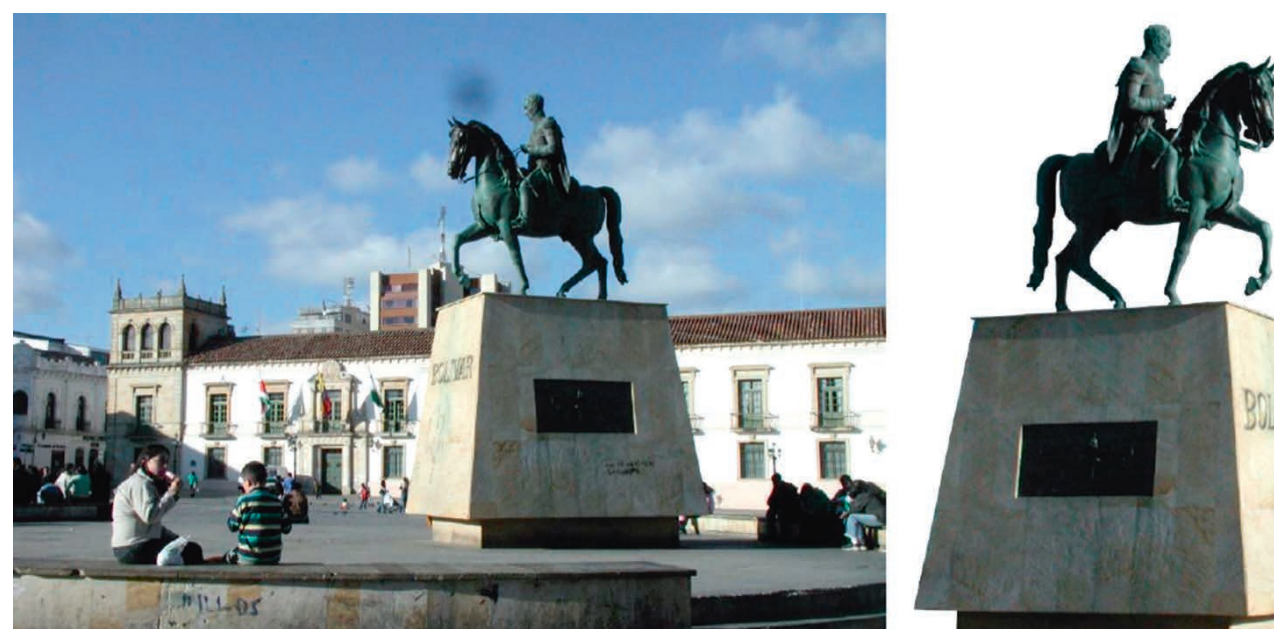

Figura 5. Recursos fotográficos. Fuente: autores. 

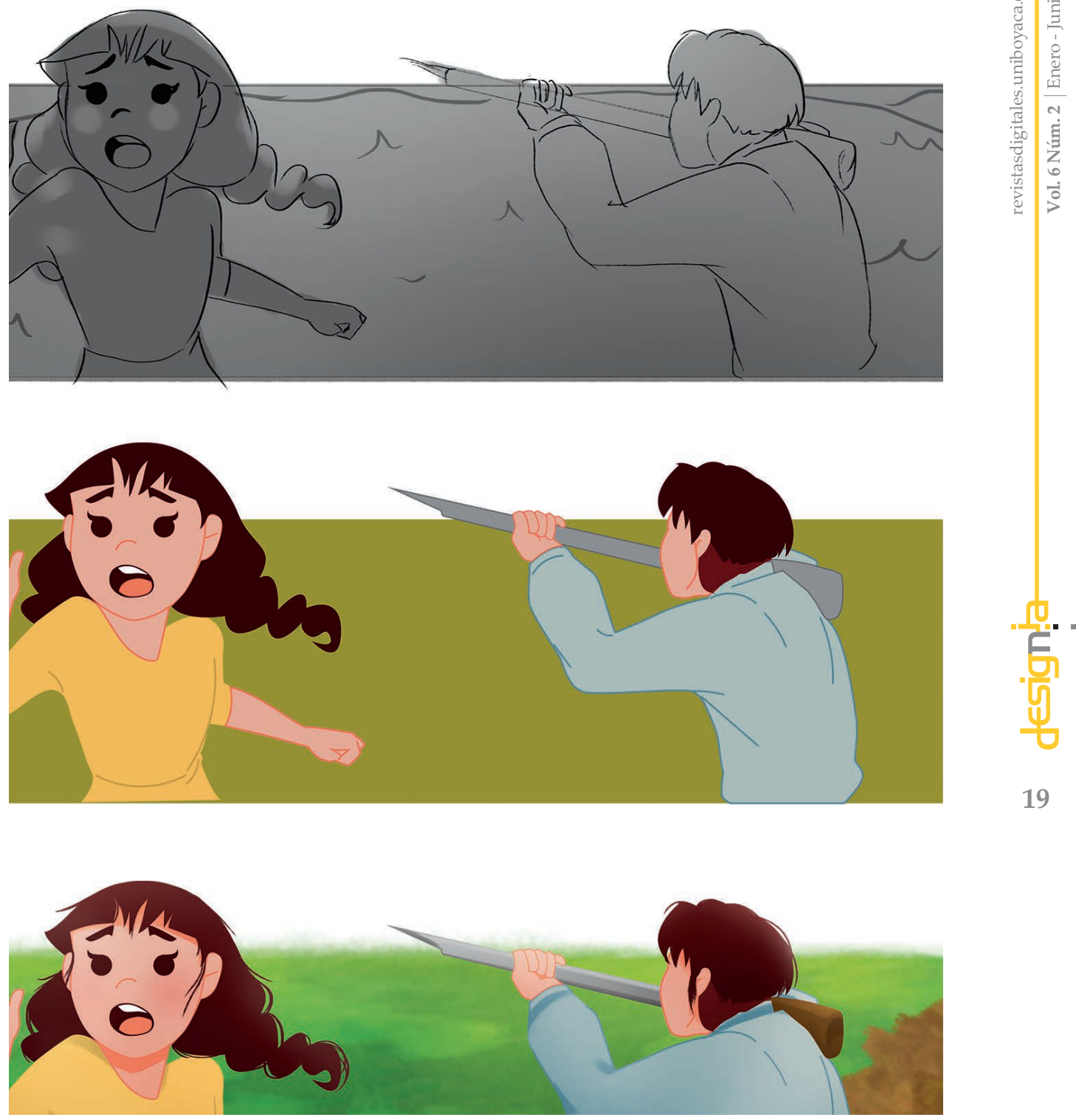

Figura 6. Proceso de ilustración.

Fuente: autores. 


\section{PROSPECTIVA Y MERCADO DE LA PROPUESTA}

Un valor que posee la propuesta es contemplar un ecosistema digital conformado por la página web donde se aloja el prototipo (e-book Puente Boyacá), la aplicación móvil que permite a los usuarios acceder de forma gratuita a los contenidos gráficos, y los audiovisuales (guía de usuario y docente, elementos de realidad aumentada y mapa de interacción para reconocer la Ruta Libertadora). Asimismo, se apunta a evidenciar el legado existente en el territorio de este memorable recorrido, con lo cual se fomenta el reconocimiento y la valoración de su patrimonio cultural y se contribuye a su divulgación, a la comunicación de su historia y de sus personajes.

Es pertinente señalar que hoy por hoy, los CED transmedia constituyen un mercado en alza, si bien para comercializarlos con éxito es preciso aplicar actividades de marketing digital. En consecuencia, además de definir como público objetivo a estudiantes entre 8 y 12 años de colegios públicos y privados (inicialmente de la región cundiboyacense) se han considerado las estrategias descritas a continuación:

\section{Elección de la tienda de aplicaciones}

La etapa de difusión y comercialización se maneja a través de las tiendas más comunes para aplicaciones móviles, como son Play Store y App Store de Apple.

\section{Difusión}

Se realiza mediante promoción impulsada en plataformas como Facebook y YouTube. Se crea material audiovisual para campañas de expectativa y difusión.

\section{Precios}

Se prevé un ecosistema digital gratuito, con una comercialización por medio de contenidos exclusivos para usuarios que no hagan parte del grupo objetivo.

\section{Empleo de canales de comunicación}

Para dar a conocer los CED transmediales es necesario utilizar distintos medios comunicativos, desde los más clásicos (notas de prensa, medios televisivos locales, páginas web institucionales, blogs corporativos, boletines o newsletters), hasta los más comunes en la actualidad (sitio web propio, aplicación móvil, redes sociales). Facebook y YouTube resultan particularmente útiles para promocionar la aplicación. 

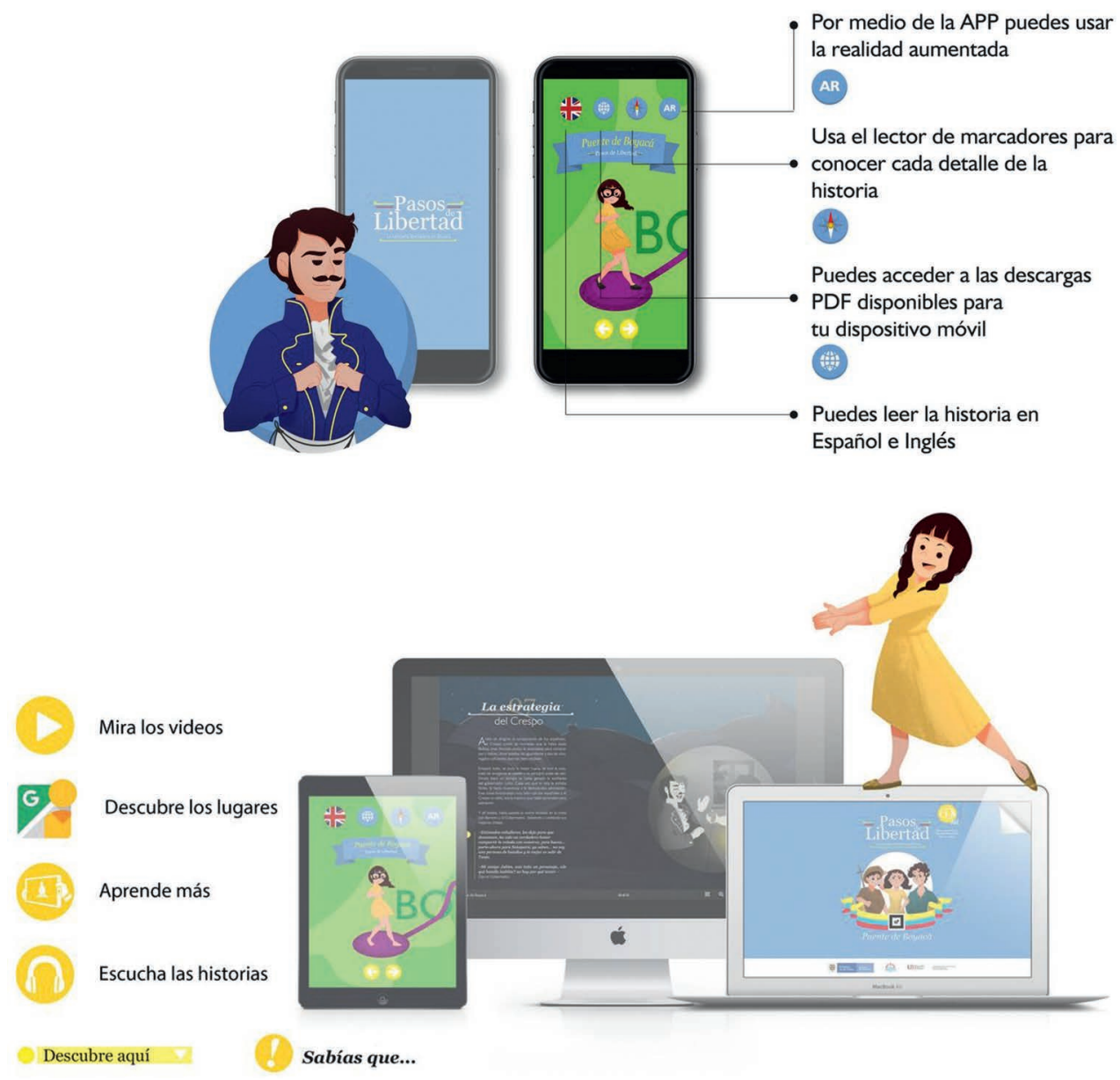

Figura 7. Caracterización gráfica de la propuesta final.

Fuente: autores.

\section{CONCLUSIONES Y EVALUACIÓN PRELIMINAR}

Como conclusión se presenta una matriz DAFO (Debilidades, Amenazas, Fortalezas y Oportunidades) con la cual se evalúa la viabilidad de la propuesta:

\section{Debilidades}

- Ante las numerosas aplicaciones existentes en el mercado, es de principal relevancia trabajar en el plan de comunicación y difusión de los CED.

- Gran parte del público objetivo puede tener dispositivos móviles que operan con software diferente al implementado en la aplicación. Inicialmente, esta se encuentra disponible como demo en la App Store de Apple. 
- Poco conocimiento del desarrollo de aplicaciones móviles. Para superar este obstáculo se sigue un trabajo interdisciplinar con otros profesionales.

- Fondos limitados para la creación, promoción y sostenibilidad del producto.

\section{Amenazas}

- Sector lleno de competidores.

- Elevado ritmo de innovación del sector.

- Mercado inmaduro y vulnerable por la aparición de nuevas tendencias tecnológicas.

- Dudas en el modelo de negocio y comercialización, ya que fue concebido para distribución gratuita a instituciones y usuarios interesados en dichas temáticas.

\section{Fortalezas}

- Es una propuesta novedosa que se sitúa entre los proyectos con aporte tecnológico y educativo en Colombia.

- Los estudiantes de los grados cuarto a sexto están familiarizados con la tecnología de los dispositivos móviles y los CED transmediales.

- El diseño digital está desarrollado para ser de uso fácil e intuitivo.

- La aplicación, el e-book y los contenidos descargables no ocupan demasiado espacio en los dispositivos. Además, el consumo de recursos es bajo.

- Los dispositivos móviles pueden funcionar en situaciones en que los usuarios disponen de baja o nula conectividad a internet.

- La difusión del producto es sencilla, pues solo se requiere acceso a internet para descargar el material de libre distribución. El resto de interacciones se da fuera de línea, lo cual abarata los costos y cualquier mantenimiento adicional.

- Los CED transmediales son fácilmente actualizables.

- La realidad aumentada proporciona un valor agregado para la divulgación.

\section{Oportunidades}

- El mercado de los CED transmediales experimenta crecimiento y posicionamiento, situación favorable para la apertura a nuevos sectores empresariales.

- Acceso a un mercado global e introducción en el sector de las aplicaciones móviles.

- Posibilidad de revolucionar el concepto de distribución de contenidos educativos.

- Al ser una aplicación gratuita, el precio no es problema para la decisión de compra.

- El material puede ser descargado e imprimido desde estaciones personales.

- Se motiva al estudiante a la lectura ya que se ofrecen nuevas formas de presentación multimedia, formatos animados, videos y material audiovisual.

- Se facilita el autoaprendizaje al ritmo de cada escolar en la medida que, desde un computador y distintos dispositivos, es posible acceder a los materiales y volver a ellos cuantas veces se requiera para leerlos y visualizarlos. 
- La propuesta es escalable, puede ser ampliada a otros contextos y adaptada a todos los sitios significativos del recorrido de la Ruta Libertadora.

Estos CED se estiman viables gracias a los recursos procedentes de la convocatoria Crea Digital 2018. Se espera que para 2019 el prototipo alcance un nivel aplicable, al menos con el grupo objetivo analizado. Igualmente, son importantes las alianzas con organizaciones privadas y públicas para acrecentar el alcance del producto final.

\section{REFERENCIAS BIBLIOGRÁFICAS}

Aguiar, B. O., Velázquez, R. M. \& Aguiar, J. L. (2019). Teacher's innovation and the use of ICTs in the higher education. Espacios, 40(2). Recuperado de https://www.revistaespacios.com/a19v40n02/19400208.html

Aparicio, G. (2005). Proyecto integrado - Polimodal. Proyecto pedagógico con modalidad a distancia para terminalidad de estudios de EGB 3 y educación polimodal Editep. Mendoza, Argentina: Editorial Universidad de Cuyo.

Cano, L. R. (2009). La difusión del patrimonio en los materiales curriculares: el caso de los gabinetes pedagógicos de Bellas Artes. Disertación Doctoral, Universidad de Málaga, España.

Carvajal, L. (2013). Luabooks. Recuperado de www.luabooks.com/nosotros

Cope, B. \& Kalantzis, M. (2009). Ubiquitous Learning. Illinois: University of Illinois.

Iriarte, F. (2011). Los niños y las familias frente a las Tecnologías de la Información y las Comunicaciones. Psicología desde el Caribe, (20), 208-224.

Martínez, H., Pinzón, J. \& Arango, N. (2017). Divulgación y enseñanza del patrimonio: interpretación de contenidos digitales y las nuevas perspectivas educativas. Designia, 5(1), 48-66. DOI: https://doi. org/10.24267/22564004.253

Mesa, M. L., Millares, R., Guerrero, Y., Jiménez, L. \& Bonilla, J. (2009). Por los caminos de la Ruta Libertadora. Tunja: Universidad de Boyacá.

Rascón, S. \& Sánchez, A. L. (2008). Las nuevas tecnologías aplicadas a la didáctica del patrimonio. Ponencia presentada en el curso: Claves para una interpretación educativa del patrimonio artístico de las ciudades, celebrado en el Centro Internacional de Estudios Históricos Cisneros (UAH) en Alcalá de Henares en Julio de 2007

Rodríguez, F. (2015). Diseño de orientación gráfica en el museo: usuarios, interfaz y experiencia. MasD, Revista Digital de Diseño, 9(16), 6-29. 